\title{
Nota Técnica: \\ Clasificación y selección de materiales de cambio de fase según sus características para su aplicación en sistemas de almacenamiento de energía térmica
}

\author{
Technical Note: \\ PCM choosing and classification according to their characteristics \\ for their application for thermal energy storage systems
}

\author{
$\underline{\text { A. Oliver }}^{(*)}$, F. J. Neila(*) A. García-Santos(*)
}

Recepción/Received: 1-III-10

Aceptación/Accepted: $24-\mathrm{X}-10$

\section{RESUMEN}

El almacenamiento térmico ha estado ligado a la edificación desde la Antigüedad, en las grandes construcciones masivas, mediante el calor sensible. Otra forma de almacenamiento térmico, que ha suscitado gran interés en las últimas décadas, es en forma de calor latente, mediante materiales de cambio de fase.

Estos materiales mantienen constante la temperatura durante el cambio de fase mientras que el material almacena o cede energía. Este hecho supone una mayor densidad energética en comparación con cualquier otro. Su uso está ligado al de las energías renovables para ajustar los periodos de suministro a los de demanda.

En este trabajo se estudian los diferentes materiales de cambio de fase, clasificándolos en diferentes grupos y analizando sus características físicas, químicas, propiedades mecánicas, térmicas, así como su compatibilidad con otros materiales, disponibilidad, precios, durabilidad... Se analizan sus usos y aplicaciones más interesantes en general.

Palabras clave: material de cambio de fase, calor latente, energía térmica, entalpía, energía renovable.

\section{SUMMARY}

Thermal energy storage has been linked to Architecture since ancient times, in large massive buildings through sensible heat. Latent heat storage -through phase change materials (PCMs) is another way of thermal storage, which has aroused great interest in recent decades.

These materials, maintain constant temperature during the phase change while the material stores or releases energy. Their energy density storage is higher than any other. Its use is linked to renewable energy to adjust the supply periods to demand.

In this paper we study the different phase change materials, classifying them and analyzing their physical, chemical, mechanical, thermal properties; availability, pricing, durability, and their compatibility with other materials. Their uses and most interesting applications are discussed.

Keywords: PCM, latent heat, thermal energy, enthalpy, renewable energy.

(*) Universidad Politécnica de Madrid (Madrid, España). 


\section{INTRODUCCIÓN}

Desde hace siglos se ha aprovechado la inercia térmica de los materiales para disminuir los consumos energéticos. El control térmico natural de los espacios habitables ha sido una práctica tradicional en todas las culturas.

En la actualidad se están llevando a cabo nuevos experimentos basados en el calor sensible de los materiales para acondicionamiento incluso de urbanizaciones. En muchos países europeos, el calor se guarda en la mayoría de las aplicaciones -en tanques de agua o lechos de la piedra - en forma de calor sensible.

Los mayores problemas de esta forma de almacenamiento térmico son la gran cantidad de masa o de volumen que se necesita para almacenar energía, y las pérdidas energéticas que se producen (vinculadas a la variación de temperatura de la masa acumuladora).

Existen otras dos formas de almacenamiento térmico desarrolladas en las últimas décadas: por reacción termoquímica y por calor latente.

Las reacciones termoquímicas poseen una elevada capacidad de almacenamiento térmico (hasta $350 \mathrm{kWh} / \mathrm{m}^{3}$ ), dependiendo de la reacción, pero para ello necesitan altas temperaturas ( $130{ }^{\circ} \mathrm{C}$ aproximadamente) (1), por lo que no se aplican con estrategias de acondicionamiento pasivo.

El calor latente es la cantidad de energía que se guarda en un material cuando cambia su estructura molecular, su fase o su estado de hidratación.

Esta forma de almacenamiento posee grandes ventajas frente a las otras:

- Gran densidad de almacenamiento -muy superior a la de calor sensible y similar a la de las reacciones termoquímicas-, por lo que se elimina la necesidad de construcción de grandes instalaciones.

- Reducción de las pérdidas energéticas derivadas de la variación de temperatura (necesaria en la forma de almacenamiento sensible).

- Posibilidad de selección de la temperatura de trabajo (proceso de carga y descarga energética del material) según las necesidades.

\section{GENERALIDADES/ANTECEDENTES}

El material de cambio de fase más conocido y utilizado es el hielo, que a $0^{\circ} \mathrm{C}$-al aplicarle energía - pasa del estado sólido a líquido sin variar la temperatura. Siendo este proceso reversible.
Desde el primer tercio del siglo pasado se han diseñado y fabricado numerosos Materiales de Cambio de Fase con diferentes propiedades físicas, químicas, cinéticas... $\mathrm{y}$, por supuesto, térmicas, para responder a diferentes necesidades, usos y aplicaciones que se les requerían.

Ya en 1932, A.A.H. Douglas inventó un aparato para almacenamiento térmico compuesto por un contenedor relleno de PCM (parafina y ácido esteárico). El calor almacenado se cargaba durante el periodo valle eléctrico.

Los siguientes estudios realizados con PCM utilizaron sales hidratadas. La doctora Maria Telkes, una de las pioneras junto a otros investigadores, logró el primer resultado práctico a finales de 1948, construyendo una casa de una planta de $135 \mathrm{~m}^{2}$, climatizada por ocho colectores solares en cubierta. El calor se almacenaba en cinco bidones llenos de sal de Glauber. En 1953 L. Gardenhire realizó un experimento similar en Las Cruces, Nuevo Méjico. En este caso se mezcló la sal de Glauber con bórax como agente nucleador. En un experimento posterior llevado a cabo en una casa solar en Princeton, New Jersey, por R. Huley (de Curtis Wright Corp.) se añadió bórax y derivado del cromo como agentes nucleadores de la Sal de Glauber para inhibir la corrosión. Tras estos tres primeros intentos fallidos, muchos investigadores han examinado muchos aspectos de los PCMs y sus aplicaciones. Posteriormente, el vehículo lunar Rover, del Apollo 15, utilizó parafina como medio de almacenamiento térmico que funcionó en tres sistemas de la misión espacial en el año 1960.

\section{TIPOS DE MATERIALES DE CAMBIO DE FASE}

De entre los posibles cambios de fase, el más interesante es el de sólido a líquido, ya que aunque la cantidad de energía almacenada es inferior que entre otros cambios de estado (sólido $\leftrightarrow$ gas), la variación de volumen es menor.

Los materiales de cambio de fase líquido-sólido más comunes en el rango de temperaturas $20^{\circ} \mathrm{C}$ y $80^{\circ} \mathrm{C}$ son las ceras de parafina, sales hidratadas, mezclas eutécticas y ácidos grasos.

En la Figura 1 se representa la clasificación de las sustancias usadas para almacenamiento térmico, según Abhat.

Las ceras de parafina están disponibles en el mercado, pero su calor latente (hasta $200 \mathrm{~kJ} / \mathrm{kg}$.) es sólo la mitad del de las sales hidratadas.

Las sales hidratadas son más baratas que las ceras de parafina y también están disponibles en el mercado, pero tienen algunas desventajas como las bajas temperaturas de fusión o la corrosión en contacto con metales. 


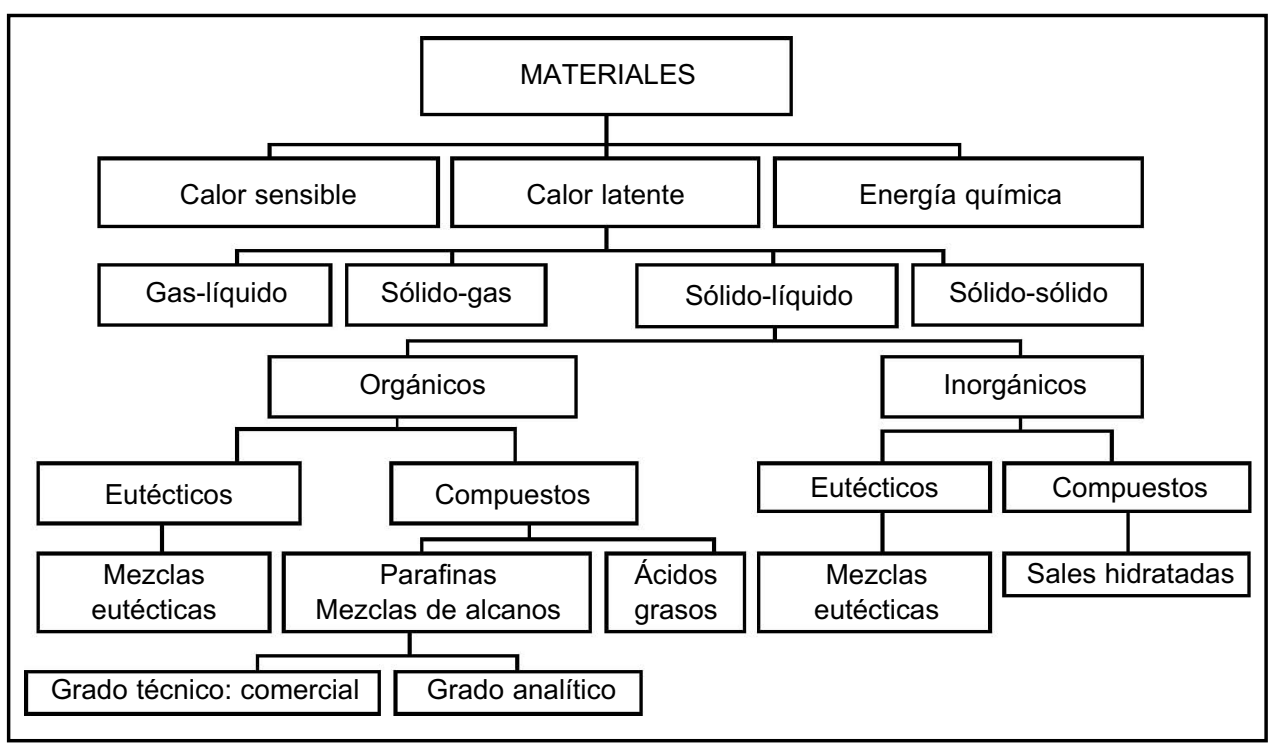

Figura 1. Clasificación de las sustancias usadas para almacenamiento térmico (4).

En las mezclas eutécticas intervienen dos componentes con punto de fusión (solidificación) más bajo que el que poseen los compuestos individualmente. Esto hace que la mezcla alcance el punto de congelación más bajo posible y ambos se solidifiquen a esa temperatura eutéctica.

Los ácidos grasos tienen, al igual que las ceras de parafina, un calor latente de aproximadamente $200 \mathrm{~kJ} / \mathrm{kg}$, pero son más caros. Abhat y Himran $(2,3)$ muestran una evaluación global muy buena de los diversos materiales de cambio de fase.

En particular, los compuestos orgánicos, en contraposición a los inorgánicos, no presentan problemas de subenfriamiento y son más estables (5). Los materiales orgánicos, especialmente sustancias como las ceras, grasas y sus ésteres, han sido recomendados como acumuladores.
Su calor latente de fusión, densidad, conductividad térmica y calor específico se encuentran en el orden de 120 $\mathrm{kJ} / \mathrm{kg}, 800 \mathrm{~kg} / \mathrm{m}^{3}, 0,20 \mathrm{~W} / \mathrm{m} \cdot \mathrm{K}$ y $1500 \mathrm{~J} / \mathrm{kg} \cdot \mathrm{K}$, respectivamente.

En la Figura 2 se representan los datos térmicos más representativos - temperatura de fusión y entalpía- de las familias químicas de materiales de cambio de fase.

\subsection{PCM inorgánicos: sales hidratadas}

Estos materiales - sales hidratadas, como la sal de Glauber- fueron muy estudiados en las primeras etapas de investigación de PCM, por su bajo coste y su capacidad de almacenamiento de grandes cantidades de calor por unidad de volumen, en comparación con las sustancias orgánicas, que las convierten en candidatos idóneos para

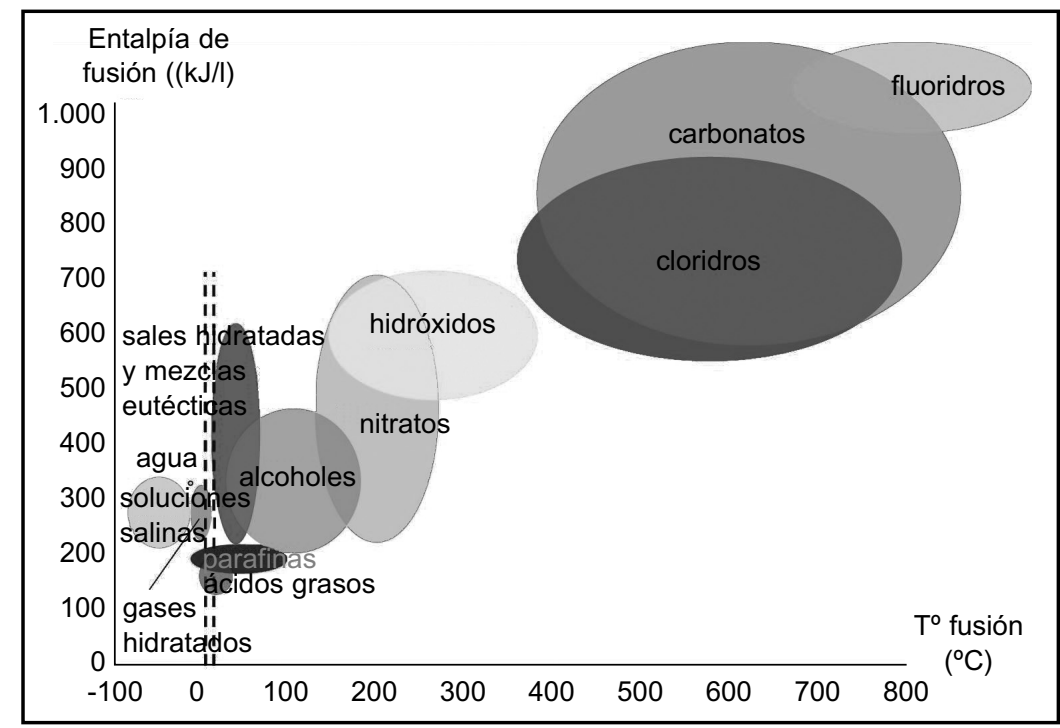

Figura 2. Muestra la relación entre la temperatura y calor de fusión para los PCM disponibles (IEA Energy Storage, Annex 17). 
sus múltiples aplicaciones en el ámbito del almacenamiento de energía solar. Lane en su trabajo (6) realiza una extensa revisión sobre los materiales de cambio de fase y en especial sobre las sales hidratadas.

Ninguna sal hidratada solidifica en su punto de congelación, aunque están subenfriadas varios grados por debajo de este. Así, el líquido subenfriado permanece en un estado metaestable. La introducción de un núcleo cristalino en el PCM provoca una cristalización espontánea de todo este.

Varios estudios se han concentrado en la búsqueda de agentes para formación de núcleos que pueden añadirse en cantidades menores al material para provocar la formación de núcleos en el PCM y eliminar así el super-enfriamiento. Se han desarrollado técnicas de encapsulado de las sales hidratadas, a través de las cuales las partículas de la emulsión, o los gránulos, o incluso grandes bloques de materiales, se encapsulan en resinas flexibles de poliéster. Para ninguna de estas técnicas se ha conseguido la completa producción, y se necesitan desarrollar muchos proyectos a futuro antes de que las sales hidratadas puedan usarse con éxito.

Nagano, Mochida et al. (7) estudiaron diferentes materiales, encontraron que el nitrato de manganeso hexahidratado puede servir de base de desarrollo de nuevos materiales de almacenamiento compuestos. El rango de fusión está entre 7,7 y $25,3^{\circ} \mathrm{C}$ y el calor de fusión es $125,0 \mathrm{~kJ} / \mathrm{kg}$. Se observó que esa sal sufre significativamente de subenfriamiento. Examinaron la influencia de varias adiciones en la variación del punto de fusión, calor de fusión y grado de subenfriamiento. Añadiendo cloruro de manganeso tetrahidratado en nitrato de manganeso hexahidratado en cantidades del $4 \%$ en peso no varía el calor de fusión. Sus propiedades térmicas le confieren un elevado potencial de seguridad y buena presentación de PCM para almacenamiento térmico en sistemas de refrigeración.

La búsqueda de sustancias con temperatura de cambio de fase próxima a la temperatura ambiente no ha encontrado buenos candidatos dentro de este grupo y las investigaciones se han dirigido más hacia las sustancias orgánicas.

Estos materiales tienen algunas propiedades atractivas como los elevados valores de calor latente, no son inflamables y su elevado contenido hídrico significa que son baratos y muy disponibles. Sin embargo, sus características de inadaptación (corrosión, inestabilidad, dificultades de resolidificación y tendencia al subenfriamiento) han conducido a la investigación de estos materiales inorgánicos, para su aplicación. Necesitan por tanto un contenedor, para utilizarlos como aditivos de otros materiales.

En la Tabla 1 se explican las ventajas e inconvenientes de los PCM inorgánicos.

Las propiedades térmicas de algunos de estos materiales se muestran en la Tabla 2.

\subsection{PCM orgánicos: parafinas, ácidos grasos y mezclas orgánicas}

Los PCM orgánicos poseen unas características que los hacen muy útiles en su aplicación en determinados elementos constructivos para almacenamiento de calor

Tabla 1

PCM inorgánicos.

\begin{tabular}{|c|c|}
\hline Ventajas & Inconvenientes \\
\hline Generalmente baratos & El encapsulado y preparación para su uso ocasionan algunos problemas* \\
\hline Densidad de almacenamiento térmico elevada & Su uso prolongado necesita de aditivos \\
\hline Elevada conductividad térmica & $\begin{array}{c}\text { Los aditivos utilizados para evitar una fusión incongruente reducen su } \\
\text { capacidad de almacenamiento latente por unidad de volumen en más } \\
\text { de un 25\% }\end{array}$ \\
\hline Temperatura de cambio de fase claramente definida & Potencialmente corrosivos con algunos metales \\
\hline No inflamables & \\
\hline
\end{tabular}

* Las sales hidratadas absorben agua fácilmente y necesitan un encapsulado semipermeable (8).

Tabla 2

Valores típicos de sales hidratadas con temperatura de fusión próximas a la temperatura de confort, para aplicación en construcción.

\begin{tabular}{|c|c|c|c|}
\hline $\mathbf{P C M}$ & Temp. fusión $\left({ }^{\circ} \mathbf{C}\right)$ & Q fusión (kJ/kg) & Referencia \\
\hline $\mathrm{KF} \cdot 4 \mathrm{H}_{2} \mathrm{O}$ & 18,5 & 231 & Nagano et al \\
\hline $\mathrm{Na}_{2} \mathrm{SO}_{4} \cdot 10 \mathrm{H}_{2} \mathrm{O}$ & 32,4 & 192 & Nagano et al \\
\hline $\mathrm{CaCl}_{2} \cdot 6 \mathrm{H}_{2} \mathrm{O}$ & $24-29$ & - & Stritih U, et al \\
\hline $\mathrm{CaCl}_{2} \cdot 6 \mathrm{H}_{2} \mathrm{O}+\mathrm{Nucleador}+\mathrm{MgCl}_{2} \cdot 6 \mathrm{H}_{2} \mathrm{O}(2: 1)$ & 23 & Cabeza et al \\
\hline
\end{tabular}


latente. Son más estables químicamente que las sustancias inorgánicas, funden y solidifican convenientemente, sin necesidad de agentes nucleadores (sustancia química para crear núcleos y formar cristales dentro del polímero) y no sufren subenfriamiento o histéresis (cuando el PCM líquido se enfría por debajo del punto de solidificación. Esto ocurre por la formación de estructuras cristalinas durante un estado termodinámicamente metaestable). No obstante, se ha demostrado que son más compatibles y más adecuados para la adsorción en varios materiales constructivos. Aunque el coste inicial de un PCM orgánico es superior al de un inorgánico, su coste es competitivo (los ácidos grasos cuestan el doble que las parafinas) según Schroeder y Gawron (9)

Sin embargo, estos materiales tienen otras desventajas. La más significativa es que son inflamables y que emiten gases nocivos durante su combustión. Otros problemas, menos extendidos, son las reacciones con los productos de la hidratación en hormigón (hidróxido de calcio), envejecimiento por oxidación, cambios de olor y volumen apreciables.

Muchos de estos inconvenientes se han eliminado. Se ha visto que el envejecimiento por oxidación de los PCM puede inhibirse utilizando un oxidante adecuado. Se está investigando también en la emisión de gases y la inflamabilidad de algunos de los PCM más efectivos, para establecer un ratio de ignición. También se está investigando sobre la compatibilidad de los PCM con el hormigón.

Se pueden resumir sus características como sigue en la Tabla 3.
Dentro de los PCM orgánicos se pueden encontrar tres grupos de sustancias diferentes: parafinas, ácidos grasos, y mezclas orgánicas.

\section{Parafinas}

Las parafinas son esencialmente un alcalino sólido refinado.

Muestran dos rangos de enfriamiento: un rango más estrecho para un breve periodo de tiempo, y un rango más extenso que ocurre durante un periodo más amplio. Ambos se interpretan como una transición de líquido a amorfo-sólida y amorfo-sólida a cristalino-sólida, respectivamente. Parte del calor latente total de fusión se almacena en la sustancia durante cada una de estas transiciones. Es importante destacar la gran diferencia entre el rango de temperatura de congelación medido experimentalmente y los datos disponibles. El resultado es de gran importancia a la hora de diseñar y operar con sistemas LTES (Latent Thermal Energy Storage, sistemas de almacenamiento de calor latente), que exige un conocimiento exacto de la temperatura de cambio de fase del material de almacenamiento térmico.

Un número importante de autores centran su trabajo en materiales orgánicos del tipo alcanos, ceras o parafinas como $(2,10-18)$. Nos han proporcionado una amplia información sobre las propiedades termofísicas de algunas parafinas y ceras.

Desde un punto de vista práctico de su aplicación en sistemas de calefacción y refrigeración pasiva, son más interesantes las parafinas comerciales y las ceras de parafina, debido a su temperatura de fusión, como puede verse en la Tabla 4. Estos materiales se producen a gran escala y se utilizan ampliamente en varios campos.

Tabla 3

Características de los PCM orgánicos.

\begin{tabular}{|c|c|}
\hline Ventajas & Inconvenientes \\
\hline Fáciles de usar & Calor latente y entalpía más bajos \\
\hline Estabilidad térmica y química & Baja conductividad térmica \\
\hline No sufren subenfriamiento & Amplio rango de fusión* \\
\hline No necesitan agente nucleador para solidificar & Grandes cambios de volumen durante el cambio de fase \\
\hline No corrosivos & Reacción potencial con hormigón \\
\hline Reciclables y ecológicamente inocuos & Potencialmente combustibles \\
\hline & Más caros \\
\hline
\end{tabular}

*Especialmente las parafinas, por las diferentes longitudes de sus cadenas de carbono.

Tabla 4

Valores típicos de energía almacenada de parafinas con temperatura de fusión próxima a la temperatura de confort (19).

\begin{tabular}{|c|c|c|c|}
\hline PCM & Temp. fusión ( $\left.^{\circ} \mathbf{C}\right)$ & Q fusión (kJ/kg) & Fuente \\
\hline Hexadecano & 18 & 236 & Darkwa K, Kim JS \\
\hline Heptadecano & 22 & 214 & Darkwa K, Kim JS \\
\hline Octadecano & 28 & 244 & Darkwa K, Kim JS \\
\hline Parafina & $20-60$ & 200 & Hawes, Feldman et al. \\
\hline RT25 & 25 & 147 & Weinlader H, et al 2005 \\
\hline
\end{tabular}




\section{Ácidos grasos}

Los ácidos grasos, derivados de productos animales y vegetales, están constituidos por una larga cadena alifática, más de 12 carbonos. Su cadena alquílica puede ser saturada o insaturada.

Su forma general es: $\mathrm{R}-\mathrm{COOH}$, donde el radical $\mathrm{R}$ es una cadena alquílica larga.

La mayoría de los ácidos grasos naturales posee un número par de átomos de carbono, esto es debido a que son biosintetizados a partir de acetato $\left(\mathrm{CH}_{3} \mathrm{CO}_{2}\right)$, el cual posee dos átomos de carbono.

En general, muestran excelentes características térmicas (punto de fusión y congelación). El rango de temperatura refrigeración es grande, y no se produce subenfriamiento, aunque en algunos casos se han detectado $(0,5 \mathrm{~K})$ Estos materiales, sin embargo, no han suscitado mucho interés en cuanto a su uso en LTES por su elevado coste, pudiéndose utilizar en dispositivos especiales de almacenamiento de calor o frío.

Estudiando los PCM alcoholes, Kakiuchi et al (20) encontraron que el eritritol es muy prometedor como PMC. Las propiedades termofísicas de los ejemplos investigados del eritritol se aportan en el Anexo A. Se trata de un elemento simple como el hielo, porque funde congruentemente. Tiene un gran calor de fusión $320 \mathrm{~kJ} / \mathrm{kg}$, casi igual que el del hielo. El problema es la elevada densidad. Hay que destacar que el eritritol cambia de volumen durante la transición de la fase sólida a la líquida. Por eso, para el intercambio de calor se necesita una estructura fuerte o un método especial para reducir el cambio de volumen. Puede observarse también subenfriamiento. Su punto de congelación es 60-100 ㄷ, Shukla, Buddhi et al. (21) han configurado el ensayo de ciclos de aceleración térmica del eritrol, con el que han observado, que se superenfría por encima de los $15^{\circ} \mathrm{C}$.

En la Tabla 5 se observa que sólo el ácido cáprico - presente en pequeñas cantidades en el aceite de coco y de palma - tiene su temperatura de fusión próxima a la de confort.

\section{Mezclas de ácidos grasos}

Las mezclas de ácidos grasos son más atractivas para fabricar y comercializar que las mezclas eutécticas. Así Feldman, Shapiro et al. (23) han investigado muchas composiciones basadas en los compuestos de ácidos grasos producidos por Henkel Canada.

Algunas mezclas con ácidos grasos desprenden un fuerte olor, por lo que se han desestimado para utilizarlos como PCM en los paneles. Algunos n-alcanos tienen cambio de fase en estado sólido. El calor de fusión del dodecano, aportado por Hong H. (24) es sustancialmente menor que el aportado por Himran S (2)

Tabla 5

Características térmicas de algunos ácidos grasos. Tm, temperatura media; Ti, temperatura interior (22).

\begin{tabular}{|c|c|c|}
\hline Mezcla (ácidos grasos) & Temp. fusión ${ }^{\circ} \mathbf{C}$ & $\Delta \mathbf{H m} \mathbf{~ k J} / \mathbf{k g}$ \\
\hline Cáprico & 31,5 & 158 \\
\hline Laurico & $42,0-44,0$ & 179 \\
\hline Pentadecano & 52 & 159 \\
\hline Mirístico & 54,0 & 190 \\
\hline Palmítico & 63,0 & 183 \\
\hline Esteárico & 70,0 & 196 \\
\hline Eritrol & $116-118$ & 126 \\
\hline
\end{tabular}

Tabla 6

Valores típicos de PCM orgánicos con temperatura de fusión próxima a la temperatura de confort (22).

\begin{tabular}{|c|c|c|c|}
\hline PCM & Temp. fusión $\left({ }^{\circ} \mathbf{C}\right)$ & Q fusión $\mathbf{( k J / k g )}$ & Ref. \\
\hline Emerest 2325 (butil estearato+butil palmitato 49/48) & $17-21$ & $138-140$ & Feldman D, Banu et al. 1996 \\
\hline Emerest2326 (butil estearato+butil palmitato 50/48) & $18-22$ & 140 & Feldman D, Banu et al. 1996 \\
\hline Butil estearato & 19 & 140 & Hawes, Feldman et al. \\
\hline Propil palmitato & 19 & 186 & Hawes, Feldman et al. \\
\hline Cáprico-laurico 82/18 & $19,1-20,4$ & 147 & Lv SL, Zhu N, Feng GH \\
\hline Cáprico-laurico 61,5/38,5 & 19,1 & 132 & Kauranen P, Peippo K \\
\hline Cáprico-laurico 45/55 & 21 & 143 & Hawes, Feldman et al. \\
\hline Cáprico-mirstico 73,5/26,5 & 21,4 & 152 & Kauranen P, Peippo K \\
\hline Cáprico-palmitato 75,2/24,8 & 22,1 & 153 & Kauranen P, Peippo K \\
\hline Peg1000+Peg600 & $23-26$ & 150,5 & Ismail KAR, Castro JNC \\
\hline Cáprico-estearato 86,6/13,4 & 26,8 & 160 & Kauranen P, Peippo K \\
\hline
\end{tabular}


Un trabajo desarrollado por Peippo K. (25) consistía en buscar un proceso que podría utilizarse para elegir un PCM con un punto de fusión predecible para ajustarse a la temperatura óptima de aplicación. Esta investigación se hizo primero con el rango de ácidos grasos. Las propiedades térmicas de varias mezclas binarias de ácidos grasos fueron investigadas para determinar el punto eutéctico de la mezcla. En este punto la mezcla se funde isotérmicamente.

La disponibilidad, los elevados calores de fusión - presentados en la Tabla 6- y la flexibilidad de ajuste de los puntos de fusión en cada mezcla para cada aplicación particular justifican su uso en los cerramientos del edificio.

Dentro de este grupo se encuentran los MCPAM (materiales con cambio de fase constituidos por aleaciones moleculares), formados por aleaciones a base de alcanos, que tienen la ventaja de ser termoajustables (26), es decir, que permiten modular la temperatura de cambio de fase a partir de su composición.

Tal y como cita Cuevas-Diarte la aleación molecular es el material orgánico lo que la aleación clásica es al metal. Se trata de una fase única, solución sólida o cristal mixto entre dos o más constituyentes. El elemento básico de la aleación es la molécula. Las diferentes moléculas ocupan aleatoriamente los nudos de una red cristalina única. Las moléculas del componente A sustituyen total o parcialmente a las moléculas del componente $B$ (en el caso de una aleación binaria). Para que la aleación sea estable, sus componentes deben responder a criterios estrictos de similitud. Expertos del REALM en Cristalografía, Termodinámica y otras disciplinas trabajan complementariamente en la definición de estos criterios: grado de homeomorfismo molecular, grado de isomorfismo cristalino, noción de familia, temperatura característica, correlaciones entre magnitudes cristalográficas y termodinámicas.

Si los componentes de una aleación presentan calores latentes de transición de fases de primer orden elevados, entonces las aleaciones moleculares elaboradas a partir de ellos presentan a su vez, propiedades muy interesantes para el almacenamiento de energía térmica. Por tanto, se trata de un material "termoajustable", que permite modular la temperatura de almacenamiento y restitución de la energía a partir de su composición. Sobre este aspecto se puede encontrar abundante información en las referencias $(27),(18,19,28,29)$.

Existe un gran número de PCM —orgánicos e inorgánicos- disponibles comercialmente. Los más interesantes para aplicarlos en construcción se exponen en la Tabla 7.

\section{CRITERIOS DE SELECCIÓN DE MATERIALES DE CAMBIO DE FASE}

Las principales características a cumplir por un PCM para almacenamiento energético son:

Tabla 7

Valores típicos de los PCM comerciales empleados en construcción.

\begin{tabular}{|c|c|c|c|c|}
\hline PCM & Tipo de producto & Temp. fusión ( $\left.^{\circ} \mathbf{C}\right)$ & Q fusión (kJ/kg) & Fuente \\
\hline Astorstat HA17 & P & $21,7-22,8$ & - & Astor wax by Honey \\
\hline RT 20 & P & 22 & 172 & Rubitherm GmBH \\
\hline- & 2 S.H. & $22-25$ & - & ZAE Bayern \\
\hline Climsel C23 & S.H. & 23 & 148 & Climator \\
\hline E23 & $*$ & 23 & 155 & (EPS) \\
\hline ClimselC24 & S.H. & 24 & 216 & Climator \\
\hline RT 25 & P & 25 & 231 & Rubitherm GmBH \\
\hline RT 26 & P & 26 & 213 & Mubitherm GmBH \\
\hline STL 27 & S.H. & 27 & 207 & Cristopia \\
\hline S27 & S.H. & 27 & 207 & Cristopia \\
\hline S27 & S.H. & 27 & - & Astor wax by Honey \\
\hline Astorstat HA18 & C & $27,2-28,3$ & 179 & Rubitherm GmBH \\
\hline RT 30 & P & 28 & 206 & Rubitherm GmBH \\
\hline RT 27 & P & 28 & 188 & Rubitherm GmbH \\
\hline RT27 & P & 28 & 188 & TEAP \\
\hline TH 29 & S.H. & 29 & 212 & TEAP \\
\hline TH29 & S.H. & 29 & 130 & Climator \\
\hline Climsel C32 & S.H. & 32 & 31 & Rubitherm GmBH \\
\hline RT32 & P & 29 &
\end{tabular}

P: parafina: S.H. Sal Hidratada: C Cera: *Mezcla de dos soluciones eutécticas no tóxicas y hielo. 


\section{Físicas}

- La densidad del líquido y del sólido deben ser similares, y preferiblemente elevadas, para poder almacenar mayor cantidad de calor por unidad de volumen.

- Variación de volumen -en el cambio de fasebaja.

\section{Cinéticas}

- Evitar el problema de histéresis, subenfriamiento o retardo al inicio de la solidificación, como ocurre en los PCM inorgánicos, que se produce al no ceder el calor latente de un PCM pese a que su temperatura sea inferior al punto de congelación.

- Poseer suficiente tasa de cristalinización: en los PCM inorgánicos se necesitan agentes nucleadores aditivos para que comience a solidificar la sustancia.

\section{Químicas}

- El proceso de cambio de fase debe ser totalmente reversible y sólo dependiente de la temperatura.

- Ser químicamente estables en el tiempo evitando su descomposición y contaminación.

- No presentar segregación de fases.

- Ser compatibles con los materiales de encapsulado y otros con los que esté en contacto: estabilidad química, para evitar oxidación, descomposición térmica, hidrólisis, corrosión y otras reacciones.

- No inflamables y resistentes al fuego.

\section{Térmicas}

- La temperatura de cambio de fase debe ser adecuada a cada aplicación particular.

- Presentar una temperatura de fusión definida o, al menos, el cambio de fase debe producirse en un valor discreto (sustancia pura) o en un pequeño intervalo de temperaturas, denominado ventana térmica (26).

- Poseer una gran capacidad de almacenamiento de calor sensible (calor específico) en estado sólido y líquido y elevada entalpía de cambio de fase, calor latente.

- Poseer una alta conductividad térmica para facilitar la transferencia de calor.

\section{Económicas}

- Estar disponible en grandes cantidades a bajo coste, para que sean viables técnica y económicamente.

- Tener un razonable ciclo de vida útil de acuerdo a su costo.

\section{CRITERIOS DE APLICACIÓN DE LOS MATERIALES DE CAMBIO DE FASE}

La utilización de PCM ha mejorado significativamente en los últimos 25 años, a lo largo de los cuales ha ido apareciendo información sobre sus características pero, aunque existe bastante información, está muy dispersa y es difícil encontrarla. Un trabajo de recopilación sobre almacenamiento de energía térmica con cambio de fase sólido-líquido ha sido desarrollado por Zalba, B (19). El trabajo se centra en materiales, transmisión de calor y sus aplicaciones, y enumera 150 materiales utilizados en investigación como materiales de cambio de fase, 45 de los cuales están disponibles comercialmente.

Las aplicaciones de las ceras de parafina han sido las más analizadas. Los resultados demostraban que la combinación de un sistema prototipo y enfriamiento nocturno proporciona un índice de almacenamiento térmico apropiado para evitar el sobrecalentamiento en condiciones habituales de verano, y que el sistema ofrece beneficios sustanciosos respecto a sistemas alternativos como las "chilled beams" y el aire acondicionado.

Las mezclas de sales hidratadas inorgánicas, agua y agentes nucleadores y estabilizadores son los PCM eutécticos usados más frecuentemente en aplicaciones de almacenamiento de frío. Pero estas mezclas eutécticas son algo complicadas de preparar y manejar, con lo que se está trabajando con otros PCM con unos materiales primarios más disponibles.

En general, los PCM tienen múltiples y variados usos, desde la electrónica (para mantener los dispositivos a una temperatura adecuada para su buen funcionamiento como en los trabajos presentados en $(26,30)$ hasta la botánica (protección térmica de plantas, para evitar heladas invernales).

También se utilizan para transporte de mercancías, sensibles a los cambios de temperaturas (material sanitario), para la industria alimentaria, industria textil (especialmente en prendas deportivas para evitar hipotermias o hipertermias)... tal y como se muestra en la Figura 3 (31)

\section{CONCLUSIONES DE MATERIALES DE CAMBIO DE FASE}

En los últimos años la utilización de PCM para almacenamiento energético ha crecido rápidamente y muchos productos basados en la técnica de PCM se han introducido en el mercado. En otras áreas se ha demostrado la viabilidad de las aplicaciones de estos productos. 


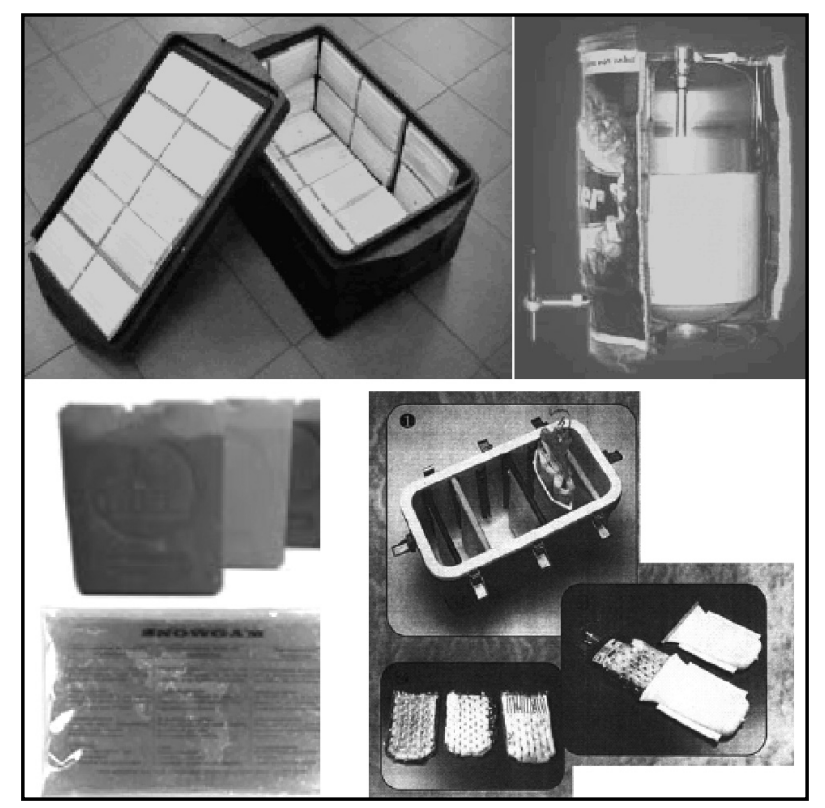

Figura 3. Ejemplos de utilización de materiales de cambio de fase: en la industria alimentaria (para conservar alimentos y bebidas), en el campo sanitario, para transporte y conservación de órganos. 1, PCM Thermal Solutions, 2 GMBH, 3-4 Sofigram, 5 Biotrans.

Nuevos PCM con características mejoradas o con propiedades físicas adecuadas a aplicaciones específicas pueden encontrarse en el mercado. En la actualidad se están desarrollando procedimientos estandarizados de medida y representación de las propiedades físicas de los PCM.

El control de temperatura utilizando PCM para transporte de productos farmacéuticos $u$ otros bienes sensibles a la temperatura ya son una tecnología habitual. La utilización de PCM para calefacción y refrigeración del cuerpo humano se ha demostrado tanto para confort personal como para aplicaciones terapéuticas. Diversas aplicaciones en diferentes campos están presentes hoy día en el mercado.
En el campo de la edificación, se han desarrollado sistemas de climatización por suelo radiante, y enfriamiento pasivo en oficinas y otros edificios, utilizando sistemas de almacenamiento energético con materiales de cambio de fase. $Y$ la utilización de la refrigeración nocturna para la refrigeración diurna, se ha demostrado en varios países. La empresa química BASF, comercializa desde hace años un panel de yeso laminado con material de cambio de fase incorporado. Una forma interesante de integración arquitectónica es la utilizada en la propuesta presentada por la UPM para el concurso Solar Decathlon 2005, donde el PCM se instalaba bajo el pavimento técnico para acondicionamiento pasivo de la vivienda (32).

\section{BIBLIOGRAFÍA}

(1) Hauer, A.: "Innovative Thermal Energy Storage Systems for Residential Use". (2002), Bavarian Center for Applied Energy Research, ZAE Bayern, p. 8.

(2) Himran, S., Suwono A.: "Characterization of Alkanes and Paraffin Waxes for Application as Phase Change Energy Storage Medium", 16 (1994 9 (Energy Sources), pp. 117-128.

(3) Abhat, A.: "Latent Heat Thermal Energy Storage in the Temperature range $20-80^{\circ} \mathrm{C}$ ". (1978) (Bericht IKE 5-209).

(4) Abhat, A.: "Low temperature latent heat thermal energy storage-Heat storage materials". Solar Energy, 30(4) (1983).

(5) Hasan, A. and Sayigh, A. A.: "Some fatty acids as phase-change thermal energy storage materials". Renewable energy, 4(1) (1994), pp. 69-76.

(6) Lane, G. A.:"Solar heat storage" Latent heat materials. Volume II. Technology. (1986): CRC Press, Inc., Boca Raton, FL.

(7) Nagano, K. et al.: "Thermal characteristics of manganese (II) nitrate hexahydrate as a phase change material for cooling systems". Applied Thermal Engineering, 23(2) (2003), pp. 229-241.

(8) Ibáñez, M. et al.: "An approach to the simulation of PCMs in building applications using TRNSYS". Applied Thermal Engineering, 25(11-12) (2005), pp 1796-1807.

(9) Schroeder, J. and Gawron, K: "Latent heat storage". International Journal of Energy Research, 5 (1981), pp. 103-109.

(10) Bardon, J. P., Vrignaud, E., Delaunay, D.: "Etude expérimentale de la fusión et de la solidification périodique d'une plaque de paraffine". Rev. Gén. Therm., Fr (1979), pp. 212-213.

(11) El-Dessouky, H. et al:: "Heat transefer in vertically aligned phase change energy storage systems". Journal of Solar Energy Engineering, Transactions of the ASME (1999), 121, pp. 98-110. 
(12) Farid, M.: "Solar Energy Storage with phase change". Energy Res, 4 (1986), pp-11.

(13) Hadjieva, M., Stoykov, R. and Filipova, T: "Composite salt-hydrate concrete system for building energy storage". Renewable Energy, 19(1-2) (2000), pp. 111-115.

(14) Kemink, R. G.: "Melting of a solid adjacent to a heated vertical cylinder with or without subcooling of the solid" (Ph. D. Thesis) (1981).

(15) Salyer Ival, O. et al.: "Advanced phase-change materials for passive solar storage applications" Final report. (1986), DOE/CE/30755T1, Dayton Univ., OH (USA). Research Inst.

(16) Samai, M., Jarny, Y. and Delaunay, D.: "An optimization method using an adjoint equation to identify solidification front location". Numerical Heat Transfer, Part B: Fundamentals, 23(1) (1993), pp. 67-89.

(17) Hawes, D., Feldman, D. and Banu, D.: "Latent heat storage in building materials". Energy and buildings, 20(1) (1993), pp. 77-86.

(18) Fieback, K., Gutberlet, H., and Buttner, D. C.: "Microwave-activated latent heat accumulator bodies" (1999).

(19) Zalba, B.: "Almacenamiento térmico de energía mediante cambio de fase. Procedimiento experimental", in Departamento de Ingeniería Mecánica. (2002), Universidad de Zaragoza, Zaragoza.

(20) Kakiuchi, H. et al.: "A study of erythritol as phase change materia"l. IEA Annex (1998), pp. 11-13.

(21) Shukla, A. et al:: "Accelerated thermal cycle test of erythritol for the latent heat storage application". Proceedings of the EM4 Indore Workshop IEA ECES IA Annex (2003), 17, pp 21-24.

(22) Kenisarin, M. and Mahkamov, K.: "Actual Problems in Using Phase-Change Materials to Store Solar Energy". NATO Advanced Study Institute Summer School on Thermal Energy Storage for Sustainable Energy Consumption (TESSEC) (2005), pp. 6-17.

(23) Feldman, D., et al.: "Fatty acids and their mixtures as phase-change materials for thermal energy storage". Solar energy materials, 18(3-4) (1989), pp. 201-216.

(24) Hong, Y. and Xin-shi, G.: "Preparation of Polyethylene-Paraffin Compound as a Form-Stable Solid-Liquid Phase Change Material", in Solar Energy Materials \& Solar Cells (2000), pp. 37-44.

(25) Peippo, K., Kauranen, P., and Lund, P.: "A Multicomponent PCM Wall Optimized for Passive Solar Heating", in Energy and Building (1991), pp. 259-270.

(26) Cuevas-Diarte, M. A., Haget, Y. and Mondieig, D.: "Nuevos materiales para el almacenamiento de energía térmica: las aleaciones moleculares". El instalador, Abril (1996), pp. 87-89.

(27) Espeau, P.: "Syncristallisation dans la famile des alcanes de C8H18 a C17H36. Condeption, elaboration et carcterisation de nouveaux materiaux a changement de phases a base d'alliages moleculaires (MACPAM). Application a la proction thermique dans le domaine agroalimentaire" (1995), Burdeos.

(28) Metivaud, V. et al.: "Solid-Solid and Solid-Liquid Equilibria in the Heneicosane-Docosane Binary System". Chem. Mater, 11(1).

(29) Roblès, L. et al:: "Mise au point sur le comportement énergétique et cristallographique des n-alcanes". II. Série de C 22 H 46 à C 27 H 56. J. Chim. Phys. 95(1), pp. 92-111 (1998).

(30) Jing, C., Martin, V. and Setterwall, F.: "The study of PCM thermal management solution for portable computer".Proc. 2nd Workshop IEA ECES IA Annex 17, Advanced Thermal Energy Storage Techniques, pp. 3-5 (2002).

(31) Salyer Ival, O. and Sircar, A. K.: "Phase change materials for heating and cooling of residential buildings and other applications". Energy Conversion Engineering Conference, IECEC-90. Proceedings of the 25th Intersociety, 4 (1990).

(32) González, F. et al.: "Phase Change Materials (PCMs) for energy storage in architecture. Use with the Magic Box prototype". Mater. Construcc. 58(291) (2008), pp. 119-126. 